\title{
Do Beta-Blockers Impact Microvolt T-Wave Alternans Testing in Patients at Risk for Ventricular Arrhythmias? A Meta-Analysis
}

\author{
PAUL S. CHAN, M.D., M.Sc., ${ }^{*}$ MICHAEL R. GOLD, M.D., Ph.D., $\dagger$ \\ and BRAHMAJEE K. NALLAMOTHU, M.D., M.P.H. $\ddagger$
}

From the *Mid-America Heart Institute and University of Missouri, Kansas City, MO; †Medical University of South Carolina, Charleston, SC; and $\ddagger$ The VA Ann Arbor Health Services Research \& Development Center of Excellence; and the University of Michigan Division of Cardiovascular Medicine, Ann Arbor, MI, USA

\begin{abstract}
Beta-Blockers and Microvolt T-Wave Alternans. Introduction: Results of microvolt T-wave alternans (MTWA) studies vary and may be influenced by whether beta-blocker therapy was withheld prior to MTWA assessment. We conducted a meta-analysis of the predictive value of MTWA screening for ventricular arrhythmic events in primary prevention patients with left ventricular dysfunction and examined whether results differed depending upon whether beta-blocker use was withheld prior to MTWA testing.
\end{abstract}

Methods and Results: Prospective studies that evaluated whether MTWA predicted ventricular arrhythmic events published between January 1980 and September 2008 were identified. Summary estimates for the predictive value of MTWA were derived with random-effects models. Nine studies involving 3,939 patients were identified. Overall, an abnormal MTWA (positive and indeterminate) test was associated with an almost 2-fold increased risk for arrhythmic events (pooled $R R=1.95,95 \%$ CI: 1.29-2.96; $P=0.002$ ). However, significant heterogeneity across studies was observed $(P=0.024)$. In the 4 studies in which betablocker therapy was not withheld prior to MTWA assessment, an abnormal MTWA test was associated with a 5-fold increased risk for arrhythmic events (pooled $R R=5.39,95 \% \mathrm{CI}$ : $2.68-10.84 ; P<0.001$ ) and was robust to sensitivity analyses. In contrast, the association was much weaker in those studies where the use of beta-blocker therapy was withheld prior to MTWA testing (pooled RR $=1.40,95 \%$ CI: 1.06-1.84; $\mathrm{P}=\mathbf{0 . 0 2}$ ).

Conclusions: In primary prevention patients with left ventricular dysfunction, the predictive power of MTWA varied widely, based on whether beta-blocker therapy was withheld prior to its assessment. This observation may explain the inconsistent results of MTWA studies in this population. $(J$ Cardiovasc Electrophysiol, Vol. 21, pp. 1009-1014, September 2010)

beta-blocker, microvolt T-wave alternans, cardiomyopathy, prognosis, sudden death

\section{Background}

Implantable cardioverter defibrillator (ICD) therapy has been shown to reduce mortality among primary prevention patients with left ventricular dysfunction. ${ }^{1,2}$ However, enthusiasm for their use has been tempered by high initial costs, device complication rates, ${ }^{3,4}$ and recent manufacturer recalls. ${ }^{5,6}$ Given these concerns and because as few as 1 in 5 patients receives a therapeutic defibrillation from their ICD ${ }^{2}$ there is great interest in further risk stratification of patients at risk for sudden cardiac death. Microvolt T-wave alternans (MTWA), which detects abnormalities in ventricular repo-

Dr. Chan is the recipient of an NIH K23 Career Development Award.

Dr. Gold has received a research grant from Cambridge Heart and serves as a consultant/advisory board member for Medtronic, Boston Scientific, and St. Jude Medical.

Other authors: No disclosures.

Address for correspondence: Paul Chan, M.D., M.Sc., Mid-America Heart Institute 5th Floor, 4401 Wornall Road, Kansas City, MO 64111. Fax: 816932-5744; E-mail: paul1chan@yahoo.com

Manuscript received 20 November 2009; Revised manuscript received 2 February 2010; Accepted for publication 8 February 2010.

doi: $10.1111 / \mathrm{j} .1540-8167.2010 .01757 . \mathrm{x}$ larization that are associated with the onset of ventricular arrhythmias, ${ }^{7,8}$ has been proposed as a method to improve the efficient use of ICD therapy by identifying patients at highest risk for sudden cardiac death. ${ }^{9,10}$

Although a prior meta-analysis found that MTWA predicts ventricular arrhythmic events among patients with ischemic and nonischemic heart failure, ${ }^{11}$ recent studies have reported less consistent results. ${ }^{12-15}$ Notably, studies of MTWA varied in their screening protocols as to whether beta-blocker therapy was withheld prior to testing, despite evidence that beta-blockers suppress MTWA amplitude and affect the presence of MTWA during testing. ${ }^{16}$ Indeed, 1 in 6 patients who screened MTWA positive when beta-blocker therapy was withheld converted to a negative test result during re-testing on beta-blocker therapy, ${ }^{16}$ and the amplitude of MTWA is significantly decreased by acute beta-blockade. ${ }^{17}$ Under ideal circumstances, a screening test for prognostication should be performed in a pharmacologic environment consistent with the patient's medical therapy to ensure that test results reflect the potential benefits of chronic drug therapy. Differences in protocols between MTWA studies regarding discontinuation of beta-blocker therapy prior to testing may therefore lead to discordant results.

Accordingly, we performed a meta-analysis to assess for heterogeneity in prognosis among MTWA studies of primary prevention patients with left ventricular dysfunction and, if present, assessed whether the ability of MTWA to predict 
ventricular arrhythmic events varied based on whether betablocker therapy was withheld prior to testing.

\section{Methods}

\section{Study Inclusion Criteria and Outcome Measures}

A systematic review of the literature was conducted to identify prospective studies that: (1) evaluated MTWA in patients with left ventricular systolic dysfunction (ejection fraction $\leq 40 \%$ ) and no prior sustained ventricular arrhythmia or cardiac arrest; (2) used a standard noninvasive MTWA screening protocol (i.e., electrophysiologic assessments of MTWA were excluded); (3) had a minimum of 6 months of follow-up and at least 100 patients in the study; (4) provided clear definitions of normal and abnormal MTWA results; and (5) provided sufficient quantitative data on the study outcome of ventricular arrhythmic events. Ventricular arrhythmic events were defined as any event involving ventricular tachycardia or fibrillation, cardiac arrest, arrhythmic death, and appropriate ICD shocks.

\section{Data Sources and Search Strategy}

We searched for published studies in the English language between January 11980 through September 302008 using PubMed, EMBASE, and all EBM Reviews (which included Cochrane Database of Systematic Reviews, Database of Abstracts and Reviews of Effects, ACP Journal Club, Cochrane Central Register of Controlled Trials, Cochrane Methodology Register, and Health Technology Assessment). The search used both key words and MeSH terms using a Boolean Search Strategy and included the following medical subject headings: prospective studies, follow-up studies, T-wave alternans, prognosis, predictive value of tests, sudden death, ventricular fibrillation, ventricular tachycardia, and ICD shocks. In addition, a hand search of bibliographies of key articles identified through the automated search was performed.

\section{Data Extraction}

Data extraction was performed by a study author (PSC) using a pre-piloted, standardized form. From each study, the following variables were abstracted: year of publication; number of study centers; study sample size; withholding of beta-blocker therapy prior to MTWA screening; etiology of left ventricular dysfunction; proportion of study patients treated with beta-blockers; proportion of patients with abnormal and normal MTWA test results; and proportion of study patients with ICDs. Information on whether beta-blocker therapy was withheld prior to MTWA screening was obtained from investigators through electronic mail when not reported in published articles. ${ }^{12,18,19}$ When results from more than one follow-up time period were reported (e.g., at 1 year and 2 years), ${ }^{15}$ we used estimates from the longer follow-up period.

\section{Statistical Analysis}

Consistent with prior work, we compared outcomes between patients with MTWA abnormal (positive and indeterminate) and normal (negative) studies. ${ }^{20,21}$ Data for the outcome of ventricular arrhythmic events were summarized using basic descriptive statistics (simple counts and proportions). A meta-analysis was conducted using a random-effects model with the restricted maximum likelihood method developed by DerSimonian and Laird. ${ }^{22}$ To evaluate for heterogeneity between studies, we calculated Cochran's Q test and $\mathrm{I}^{2}$, which represents the degree of inconsistency among studies. We focused on unadjusted results to be consistent, because not all studies performed multivariable analyses. One study reported no events in patients with normal MTWA, ${ }^{18}$ and so we assigned a non-zero $(0.5)$ correction factor to calculate its relative risk ratio. ${ }^{23}$ Publication bias was evaluated using the Begg's test.

Since we hypothesized a priori that withholding betablocker therapy prior to MTWA testing would lead to significant heterogeneity across the trials, we report our findings stratified by this category using subgroup analyses. Additional subgroup analyses were conducted to explore the heterogeneity in risk ratios between studies based upon other study criteria, which included: proportion of study patients with ICDs (low $[<40 \%]$ vs high $[\geq 40 \%]$ ); time period of publication (before vs since 2006); number of study centers ( $<10$ vs $\geq 10$ ); and etiology of left ventricular dysfunction (ischemic, nonischemic, or both). Finally, we performed sensitivity analyses to examine the influence of each study on the pooled estimate by omitting each study one at a time. All statistical tests were 2-sided and were evaluated at a significance level of 0.05. STATA version 10.0 (College Station, TX, USA) was used to conduct all analyses.

\section{Results}

Nine studies involving 3,939 patients met study inclusion criteria. ${ }^{10,12-15,18,19,24,25}$ Of these, 4 conducted MTWA testing on beta-blocker therapy, while 5 withheld beta-blocker therapy for at least 24 hours prior to testing (Table 1). Four of the studies were conducted among patients with left ventricular dysfunction due to an ischemic etiology, 3 were among patients with a nonischemic etiology, and 2 were among patients with either an ischemic or nonischemic etiology. Six of the studies were multicentered trials, while 3 were single institution studies.

Results from the individual studies are summarized in Table 2. Overall, 5 studies found an association between an abnormal MTWA test and increased risk for ventricular arrhythmic events. ${ }^{10,14,18,19,25}$ Individual study hazard ratios (HRs) for the 9 studies ranged from 1.11 (95\% confidence interval [CI]: $0.63-1.95)$ to 6.53 (95\% CI: 2.35-18.11). When the results from all 9 studies were pooled, an abnormal MTWA result was associated with a 2-fold increased risk for ventricular arrhythmic events (pooled risk ratio [RR] of 1.95, 95\% CI: 1.29-2.96; $\mathrm{P}=0.002)$. However, significant heterogeneity was present in the overall pooled estimate $(\mathrm{P}=$ 0.024).

Because of the heterogeneity across studies, we stratified our analyses based on our a priori criteria of whether betablocker therapy was withheld prior to MTWA assessment. Among studies where the use of beta-blockers was continued during MTWA testing, ${ }^{14,18,19,25}$ an abnormal MTWA test was associated with a greater than 5-fold increased risk for ventricular arrhythmic events (pooled RR of $5.39,95 \% \mathrm{CI}$ : 2.68-10.84; $\mathrm{P}<0.001$ ) (Fig. 1). This result was robust to sensitivity analyses when each study was systematically eliminated one at a time. In contrast, the association was much weaker in those studies where the use of beta-blocker therapy 
TABLE 1

Characteristics of Prospective Studies of Microvolt T-Wave Alternans in Primary Prevention Patients with Left Ventricular Dysfunction

\begin{tabular}{|c|c|c|c|c|c|c|c|c|}
\hline Study & $\begin{array}{c}\text { Year of } \\
\text { Publication }\end{array}$ & $\begin{array}{l}\text { Number of } \\
\text { Centers }\end{array}$ & $\begin{array}{c}\text { Population } \\
\text { Type }\end{array}$ & $\begin{array}{c}\text { Number } \\
\text { of } \\
\text { Patients }\end{array}$ & $\begin{array}{c}\text { Mean } \\
\text { Follow-Up } \\
\text { (months) }\end{array}$ & $\begin{array}{c}\text { Beta-Blocker } \\
\text { Withheld Prior to } \\
\text { Testing? }\end{array}$ & $\begin{array}{c}\text { Proportion on } \\
\text { on Beta- } \\
\text { Blockers, \% }\end{array}$ & $\begin{array}{c}\text { Proportion } \\
\text { with } \\
\text { ICDs, \% }\end{array}$ \\
\hline Klingenheben ${ }^{18}$ & 2000 & 1 & Ischemic & 107 & 15 & No & Not reported & 0 \\
\hline Hohnloser ${ }^{19}$ & 2003 & 1 & Nonischemic & 137 & 14 & No & Not reported & 27 \\
\hline Grimm $^{24}$ & 2003 & 1 & Nonischemic & 263 & 52 & Yes & 73 & 0 \\
\hline Bloomfield $^{25}$ & 2006 & 11 & Both & 587 & 24 & No & 81 & 12 \\
\hline Chow $^{10}$ & 2006 & 7 & Ischemic & 768 & 18 & Yes & 82 & 51 \\
\hline ALPHA $^{14}$ & 2007 & 9 & Nonischemic & 446 & 19 & No & 80 & 8 \\
\hline SCD-HeFT ${ }^{13}$ & 2008 & 37 & Both & 490 & 35 & Yes & 74 & 34 \\
\hline MASTER $^{12}$ & 2008 & 50 & Ischemic & 575 & 26 & Yes & 87 & 100 \\
\hline $\mathrm{ABCD}^{15}$ & 2009 & 42 & Ischemic & 566 & 24 & Yes & 86 & 87 \\
\hline
\end{tabular}

$\mathrm{ICD}=$ implantable cardioverter defibrillator.

was withheld prior to MTWA testing ${ }^{10,12,13,15,24}$ (pooled RR of $1.40,95 \%$ CI: $1.06-1.84 ; \mathrm{P}=0.02$; Fig. 1 ) and was not robust to sensitivity analysis when a key study ${ }^{10}$ was eliminated (pooled $\mathrm{RR}=1.27,95 \% \mathrm{CI}: 0.96-1.68 ; \mathrm{P}=0.10$ ). In both subgroups, stratifying results by whether beta-blocker therapy was withheld prior to MTWA assessment eliminated the presence of statistical heterogeneity in the pooled analyses ( $\mathrm{P}$ for heterogeneity $=0.84$ for studies not withholding beta-blockers and $\mathrm{P}$ for heterogeneity $=0.38$ for studies withholding beta-blockers). Notably, all 4 studies that did not find an increased risk of ventricular arrhythmic events with an abnormal MTWA result had withheld use of beta-blocker therapy prior to MTWA testing. ${ }^{12,13,15,24}$

The sensitivity, specificity, positive predictive value, and negative predictive value (NPV) of MTWA testing for each individual study and stratified by whether beta-blocker use was withheld prior to MTWA testing are depicted in Table 3. In the 5 studies where beta-blocker therapy was withheld prior to testing, MTWA screening yielded an overall sensitivity of $77 \%$ and an NPV of $91 \%$ for predicting ventricular arrhythmic events during follow-up. In contrast, in the 4 studies where beta-blocker therapy was continued during testing, MTWA screening yielded an overall sensitivity of $92 \%$ and an NPV of $98 \%$.

Finally, subgroup analyses found that use of beta-blocker therapy during MTWA assessment was the variable that best explained the statistical heterogeneity in prognosis among the MTWA studies (Table 4). Most notably, the pooled RR among studies with a low $(<40 \%)$ proportion of patients with ICDs was not substantially different among studies with a high ( $\geq 40 \%)$ proportion of patients with ICDs.

\section{Discussion}

We found a significant association between an abnormal MTWA test and increased risk for ventricular arrhythmic events among primary prevention patients with left ventricular dysfunction in the current literature, but also noted significant heterogeneity across these trials. Notably, studies continuing use of beta-blocker therapy during MTWA testing showed a stronger and more consistent 5-fold increased risk for ventricular arrhythmic events, whereas a much weaker relationship was observed among studies withholding betablocker therapy prior to MTWA testing.

In prior work, administration of beta-blocker therapy at the time of MTWA testing was found to significantly reduce the amplitude of MTWA. ${ }^{16,17}$ One in 6 patients with a positive MTWA test off beta-blockers converted to a normal test result upon rescreening on beta-blocker therapy, as their maximal T-wave amplitude had decreased below the threshold of $1.9 \mathrm{mV}$ for a positive test. ${ }^{16}$ Another study found that 1 in 2 patients with a positive MTWA result converted to a normal

\section{TABLE 2}

Results of Prospective Studies of Microvolt T-Wave Alternans in Primary Prevention Patients with Left Ventricular Dysfunction

\begin{tabular}{|c|c|c|c|c|c|c|c|}
\hline \multirow[b]{2}{*}{ Study } & \multicolumn{3}{|c|}{ MTWA Abnormal } & \multicolumn{3}{|c|}{ MTWA Normal } & \multirow{2}{*}{$\begin{array}{c}\text { Hazard Ratio } \\
(95 \% \mathrm{CI})\end{array}$} \\
\hline & $\mathbf{N}$ & Event & No Event & $\mathbf{N}$ & Event & No Event & \\
\hline Klingenheben ${ }^{18}$ & 74 & 13 & 61 & 33 & 0 & 33 & Undefined $\dagger$ \\
\hline Hohnloser ${ }^{19}$ & 103 & 16 & 87 & 34 & 2 & 32 & $3.44(0.03-459.0)$ \\
\hline Grimm $^{24}$ & 191 & 31 & 160 & 72 & 7 & 65 & $1.30(0.59-2.90)$ \\
\hline Bloomfield $^{25}$ & 360 & 51 & 309 & 189 & 4 & 185 & $6.53(2.35-18.11)$ \\
\hline Chow $^{10}$ & 514 & 57 & 457 & 254 & 11 & 243 & $2.93(1.33-6.46)$ \\
\hline ALPHA $^{14}$ & 292 & 29 & 263 & 154 & 4 & 150 & $4.01(1.41-11.41)$ \\
\hline SCD-HeFT ${ }^{13}$ & 355 & 59 & 296 & 135 & 16 & 119 & $1.11(0.63-1.95)$ \\
\hline MASTER $^{12}$ & 361 & 48 & 313 & 214 & 22 & 192 & $1.26(0.76-2.09)$ \\
\hline $\mathrm{ABCD}^{15}$ & 401 & 49 & 352 & 165 & 16 & 149 & $1.4(0.8-2.2) \ddagger$ \\
\hline
\end{tabular}

MTWA = microvolt T-wave altnernans.

$\dagger$ Hazard ratio undefined as there were no events in the MTWA normal group.

$\ddagger$ Two-year outcomes obtained from study authors. 


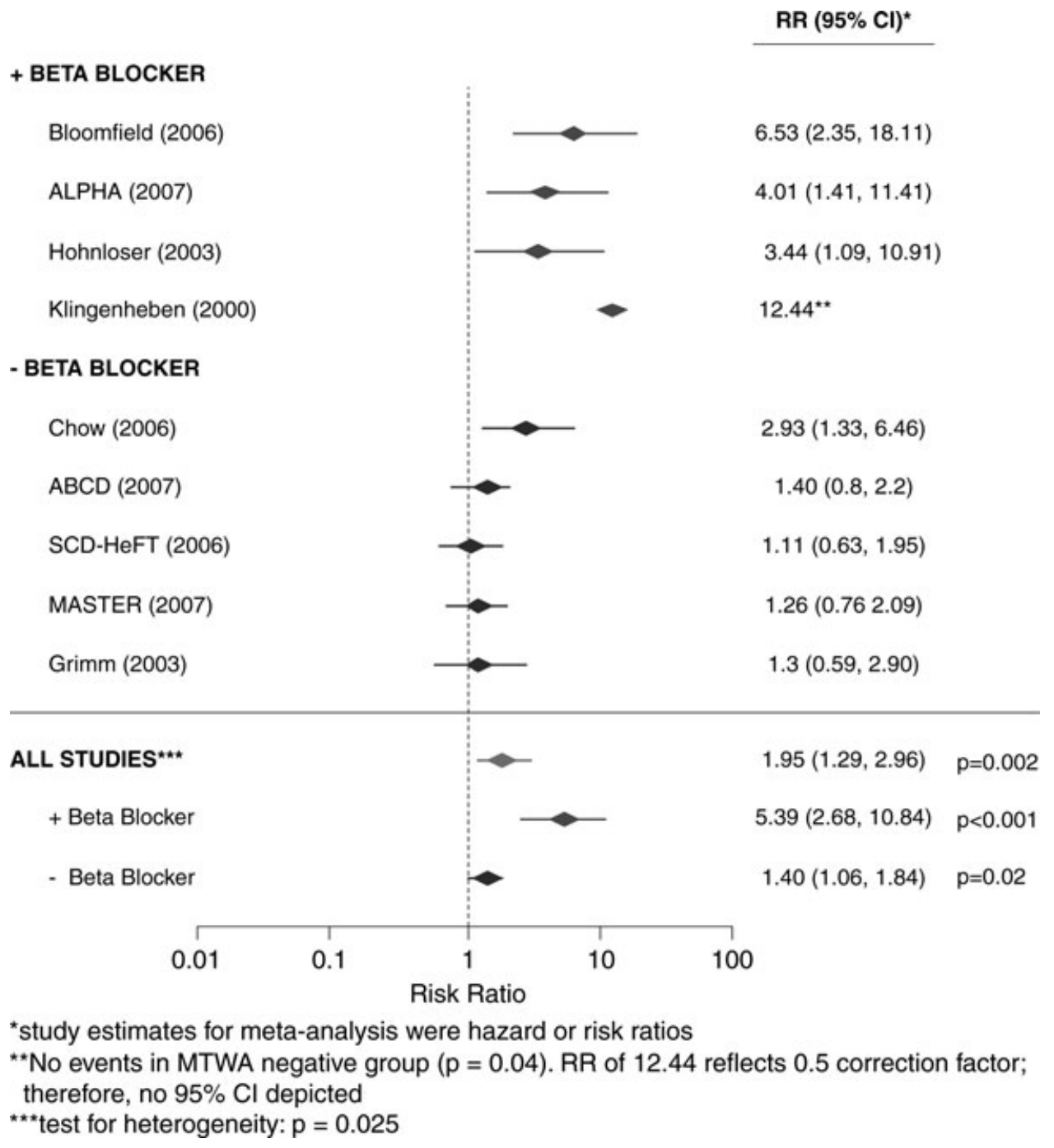

Figure 1. Association between MTWA and ventricular arrhythmic events, stratified by screening protocol discontinuation of beta-blocker therapy. Significant heterogeneity was observed among studies $(P=0.025)$. Subgroup analyses found a strong, consistent, 5-fold increased for ventricular arrhythmic events in studies where beta-blocker therapy was not discontinued prior to MTWA assessment. In contrast, a weak association between an abnormal MTWA test and risk for arrhythmic events was observed in studies that withheld beta-blocker therapy prior to MTWA assessment.

test result upon administration of beta-blocker therapy. ${ }^{17} \mathrm{~A}$ third study found that beta-blocker administration resulted in a negative MTWA test result in 8 of $26(30.8 \%)$ patients who initially screened positive. ${ }^{26}$ Because beta-blockers may alter the development of MTWA results, they may also modulate susceptibility to ventricular arrhythmias. Indeed, it has been argued that MTWA testing should be performed on betablocker therapy to provide a risk stratification that more reliably reflects the 'pharmacologic milieu' of the patient. ${ }^{16} \mathrm{By}$ performing MTWA screening off beta-blockers, prior studies may have measured higher MTWA amplitudes than when on beta-blockers and may have classified a certain proportion of otherwise MTWA negative patients as positive (i.e., falsepositives). As a result, the predictive power of MTWA for ventricular arrhythmic events in studies where beta-blocker therapy was withheld prior to testing may have been substantially diluted and could account for the wide variation in MTWA study results. This issue deserves further investigation and suggests that future trials should consider assessing MTWA both on and off beta-blockers to confirm the findings of this study.
The rationale for withholding beta-blocker therapy prior to MTWA screening has been to decrease the proportion of indeterminate results, as a negative result requires achieving heart rates of 110-120 beats per minute and documentation of no sustained MTWA. ${ }^{27}$ However, recent studies found that indeterminate MTWA tests have similar prognostic utility as positive tests, ${ }^{20,21}$ even among patients unable to achieve this heart rate level. This suggests that the protocol requirement of withholding beta-blocker therapy prior to screening may be unnecessary, especially if doing so affects the predictive power of MTWA.

In a recent meta-analysis, Hohnloser et al. ${ }^{28}$ called into question the validity of including ICD shocks as a clinical endpoint in MTWA studies. The authors found that the predictive power of an abnormal MTWA test result was excellent among studies with low ICD use and mediocre among studies with high ICD use. However, in this meta-analysis, we did not find that the predictive power of an abnormal MTWA test result differed substantially by ICD use. Our results likely differed from the Hohnloser meta-analysis because our study: (1) included only patients with left ventricular systolic 


\section{TABLE 3}

Summary of Study Accuracy, Stratified by Whether Beta-Blocker Therapy Was Withheld Prior to Microvolt T-Wave Alternans Screening

\begin{tabular}{|c|c|c|c|c|}
\hline Study & Sensitivity, \% & Specificity, \% & PPV, \% & NPV, \% \\
\hline \multicolumn{5}{|c|}{ Screening on beta-blockers } \\
\hline Bloomfield $^{25}$ & 93 & 37 & 14 & 98 \\
\hline ALPHA $^{14}$ & 88 & 36 & 10 & 97 \\
\hline Hohnloser ${ }^{19}$ & 89 & 27 & 16 & 94 \\
\hline Klingenheben ${ }^{18}$ & 100 & 35 & 18 & 100 \\
\hline SUMMARY & 92 & 36 & 13 & 98 \\
\hline \multicolumn{5}{|c|}{ Screening off beta-blockers } \\
\hline Chow ${ }^{10}$ & 84 & 35 & 11 & 96 \\
\hline $\mathrm{ABCD}^{15}$ & 75 & 30 & 12 & 90 \\
\hline SCD-HeFT ${ }^{13}$ & 79 & 29 & 17 & 88 \\
\hline MASTER $^{12}$ & 69 & 38 & 13 & 90 \\
\hline Grimm $^{24}$ & 82 & 29 & 16 & 90 \\
\hline SUMMARY & 77 & 33 & 13 & 91 \\
\hline
\end{tabular}

Studies continuing beta-blocker therapy during microvolt T-wave alternans testing were associated with higher sensitivity and NPV for ventricular arrhythmic events. NPV $=$ negative predictive value; $\mathrm{PPV}=$ positive predictive value.

dysfunction; (2) excluded patients with invasive assessments of MTWA; and (3) categorized the Sudden Cardiac Death in Heart Failure Trial (SCD-HeFT) as a low ICD use study, as only $34 \%$ of patients in the MTWA substudy of SCD-HeFT received an ICD.

While an abnormal MTWA was associated with a greater than 5-fold increased risk for ventricular arrhythmic events in studies where beta-blocker therapy was continued during screening, simultaneously demonstrating that patients with normal test results have a low event rate is critical in order for MTWA to be considered for optimal risk stratification of primary prevention patients for ICD therapy. Indeed, we found that the NPV was $98 \%$ for an abnormal MTWA test result in studies where beta-blocker therapy was continued during

\section{TABLE 4}

Results of All Subgroup Analyses

\begin{tabular}{lcc}
\hline Stratified By & $\begin{array}{c}\text { Studies } \\
\text { (Number) }\end{array}$ & $\begin{array}{c}\text { Pooled Risk } \\
\text { Ratio (95\% CI) }\end{array}$ \\
\hline $\begin{array}{l}\text { Beta-blocker use } \\
\quad \text { Continued during MTWA screening }\end{array}$ & 4 & $5.39(2.68-10.84)$ \\
$\quad$ Withheld prior to MTWA screening & 5 & $1.40(1.06-1.84)$ \\
$\begin{array}{l}\text { Proportion of study patients with ICDs } \\
\quad<40 \%\end{array}$ & 6 & $2.55(1.18-5.52)$ \\
$\quad \geq 40 \%$ & 3 & $1.58(1.03-2.43)$ \\
Number of study sites & & \\
$\quad<10$ & 5 & $2.51(1.42-4.43)$ \\
$\quad \geq 10$ & 4 & $1.64(0.95-2.81)$ \\
Publication year & 3 & $2.02(0.59-6.95)$ \\
$\quad$ Before 2006 & 6 & $2.01(1.24-3.26)$ \\
$\quad$ Since 2006 & & \\
Etiology of cardiomyopathy & 4 & $1.71(1.05-2.79)$ \\
$\quad$ Ischemic & 3 & $2.14(0.90-5.07)$ \\
$\quad$ Nonischemic & $2.55(0.45-14.45)$ \\
\hline Ischemic and nonischemic & 2 & 2.55
\end{tabular}

The heterogeneity in MTWA studies was best explained by whether betablocker therapy was withheld prior to MTWA screening. The pooled risk ratios were not as different from one another when studies were stratified by the proportion of study patients with implantable cardioverter defibrillators (ICD), number of enrolled sites, publication year, or the etiology of left ventricular dysfunction. $\mathrm{CI}=$ confidence interval. screening. Based on our prior economic model of MTWA screening, ${ }^{29}$ a strategy of implanting ICDs in all eligible patients, as compared with a strategy of implanting ICDs only in patients identified as high-risk when the RR is 5 with an abnormal MTWA test, would yield an incremental costeffectiveness ratio of $\sim$ US $\$ 150,000$ per quality-adjusted lifeyear and would not be considered cost-effective. However, future studies are needed to confirm the accuracy of MTWA test results when beta-blocker therapy is not withheld prior to screening before endorsement of their routine use in risk stratification.

Our study should be interpreted in the context of several limitations. We did not have patient-level data to conduct an on-treatment analysis or a multivariable analysis. Additionally, definitions for the study endpoint of ventricular arrhythmic events varied across studies. We were unable to examine the association between MTWA and either mortality or appropriate ICD shocks, as these were not routinely reported in many studies. Moreover, because we did not have patient-level data, we were unable to determine whether some of the heterogeneity across MTWA studies was due to inclusion of appropriate ICD shocks as part of a combined arrhythmic endpoint in more recent studies. We were unable to assess the impact of withholding other medications with antiadrenergic and antiarrhythmic effects (e.g., Vaughan Williams Class III agents) prior to MTWA screening. However, the majority of patients with left ventricular dysfunction are not treated with conventional antiarrhythmic therapy. Finally, while prior studies only examined the acute effect of intravenous beta-blockers on MTWA amplitude, it is unclear whether oral beta-blockers would have similar effects.

In conclusion, an abnormal MTWA test was associated with a 5-fold increased risk for ventricular arrhythmic events among primary prevention patients with left ventricular dysfunction in studies where beta-blocker therapy was continued during MTWA screening, while a weak association was seen in studies where beta-blocker therapy was withheld prior to screening. This observation may help explain the inconsistent results of MTWA studies in this population.

\section{References}

1. Moss AJ, Zareba W, Hall WJ, Helmut K, Wilber DJ, Cannom DS, Daubert JP, Higgins SL, Brown MW, Andrews ML: Prophylactic implantation of a defibrillator in patients with myocardial infarction and reduced ejection fraction. N Engl J Med 2002;346:877-883.

2. Bardy GH, Lee KL, Mark DB, Poole JE, Packer DL, Boineau R, Domanski M, Troutman C, Anderson J, Johnson G, McNulty SE, ClappChanning N, Davidson-Ray LD, Fraulo ES, Fishbein DP, Luceri RM, Ip JH: Amiodarone or an implantable cardioverter-defibrillator for congestive heart failure. N Engl J Med 2005;352:225-237.

3. Kleemann T, Becker T, Doenges K, Vater M, Senges J, Schneider S, Saggau W, Weisse U, Seidl K: Annual rate of transvenous defibrillation lead defects in implantable cardioverter-defibrillators over a period of $>10$ years. Circulation 2007;115:2474-2480.

4. Maisel WH: Safety issues involving medical devices: Implications of recent implantable cardioverter-defibrillator malfunctions. JAMA 2005;294:955-958

5. Maisel WH, Moynahan M, Zuckerman BD, Gross TP, Tovar OH, Tillman DB, Schultz DB: Pacemaker and ICD generator malfunctions: Analysis of Food and Drug Administration annual reports. JAMA 2006;295:1901-1906.

6. Maisel WH, Sweeney MO, Stevenson WG, Ellison KE, Epstein LM: Recalls and safety alerts involving pacemakers and implantable cardioverter-defibrillator generators. JAMA 2001;286:793799 . 
7. Lab MJ, Lee JA: Changes in intracellular calcium during mechanical alternans in isolated ferret ventricular muscle. Circ Res 1990;66:585595.

8. Armoundas AA, Tomaselli GF, Esperer HD: Pathophysiological basis and clinical application of T-wave alternans. J Am Coll Cardiol 2002;40:207-217.

9. Bloomfield DM, Steinman RC, Namerow PB, Parides M, Davidenko J, Kaufman ES, Shinn T, Curtis A, Fontaine J, Holmes D, Russo A, Tang C, Bigger JT: Microvolt T-wave alternans distinguishes between patients likely and patients not likely to benefit from implanted cardiac defibrillator therapy: A solution to the Multicenter Automatic Defibrillator Implantation Trial (MADIT) II conundrum. Circulation 2004;110:1885-1889.

10. Chow T, Kereiakes DJ, Bartone C, Booth T, Schloss EJ, Waller T, Chung E, Menon S, Nallmothu BK, Chan PS: Prognostic utility of microvolt T-wave alternans in risk stratifying patients with ischemic cardiomyopathy. J Am Coll Cardiol 2006;47:1820-1827.

11. Gehi AK, Stein RH, Metz LD, Gomes JA: Microvolt T-wave alternans for the risk stratification of ventricular tachyarrhythmic events: A metaanalysis. J Am Coll Cardiol 2005;46:75-82.

12. Chow T, Kereiakes DJ, Onufer J, Woelfel A, Gursoy S, Peterson BJ, Brown ML, Pu W, Benditt DG: Does microvolt T-wave alternans testing predict ventricular tachyarrhythmias in patients with ischemic cardiomyopathy and prophylactic defibrillators? The MASTER (Microvolt T Wave Alternans Testing for Risk Stratification of Post-Myocardial Infarction Patients) trial. J Am Coll Cardiol 2008;52:1607-1615.

13. Gold MR, Ip JH, Costantini O, Poole JE, McNulty S, Mark DB, Lee KL, Bardy GH: Role of microvolt T-wave alternans in assessment of arrhythmia vulnerability among patients with heart failure and systolic dysfunction: Primary results from the T-wave alternans sudden cardiac death in heart failure trial substudy. Circulation 2008;118:2022-2028.

14. Salerno-Uriarte JA, De Ferrari GM, Klersy C, Pedretti RF, Tritto M, Sallusti L, Libero L, Pettinati G, Molon G, Cumis A, Occhetta E, Morandi F, Ferrero P, Accardi F: Prognostic value of T-wave alternans in patients with heart failure due to nonischemic cardiomyopathy: Results of the ALPHA Study. J Am Coll Cardiol 2007;50:1896-1904.

15. Costantini O, Hohnloser SH, Kirk MM, Lerman BB, Baker JH, Sethuraman B, Dettmer MM, Rosenbaum DS: The ABCD (Alternans Before Cardioverter Defibrillator) Trial: Strategies using T-wave alternans to improve efficiency of sudden cardiac death prevention. J Am Coll Cardiol 2009;53:471-479.

16. Klingenheben T, Gronefeld G, Li YG, Hohnloser SH: Effect of metoprolol and d,1-sotalol on microvolt-level T-wave alternans. Results of a prospective, double-blind, randomized study. J Am Coll Cardiol 2001;38:2013-2019.

17. Rashba EJ, Cooklin M, MacMurdy K, Kavesh N, Kirk M, Sarang S, Peters RW, Shorofsky SR, Gold MR: Effects of selective autonomic blockade on T-wave alternans in humans. Circulation 2002;105:837842 .

18. Klingenheben T, Zabel M, D'Agostino RB, Cohen RJ, Hohnloser SH: Predictive value of T-wave alternans for arrhythmic events in patients with congestive heart failure. Lancet 2000;356:651652 .

19. Hohnloser SH, Klingenheben T, Bloomfield D, Dabbous O, Cohen RJ: Usefulness of microvolt T-wave alternans for prediction of ventricular tachyarrhythmic events in patients with dilated cardiomyopathy: Results from a prospective observational study. J Am Coll Cardiol 2003;41:2220-2224.

20. Chan PS, Bartone C, Booth T, Kereiakes D, Chow T: Prognostic implication of redefining indeterminate microvolt $\mathrm{T}$-wave alternans studies as abnormal or normal. Am Heart J 2007; 153:523-529.

21. Kaufman ES, Bloomfield DM, Steinman RC, Namerow PB, Costantini O, Cohen RJ, Bigger JT: "Indeterminate" microvolt T-wave alternans tests predict high risk of death or sustained ventricular arrhythmias in patients with left ventricular dysfunction. J Am Coll Cardiol 2006;48:1399-1404.

22. DerSimonian R, Laird N: Meta-analysis in clinical trials. Controlled clinical trials 1986;7:177-188.

23. Diamond GA, Bax L, Kaul S: Uncertain effects of rosiglitazone on the risk for myocardial infarction and cardiovascular death. Ann Intern Med 2007; 147:578-581.

24. Grimm W, Christ M, Bach J, Muller HH, Maisch B: Noninvasive arrhythmia risk stratification in idiopathic dilated cardiomyopathy: Results of the Marburg Cardiomyopathy Study. Circulation 2003; 108:2883-2891.

25. Bloomfield DM, Bigger JT, Steinman RC, Namerow PB, Parides MK, Curtis AB, Kaufman ES, Davidenko JM, Shinn TS, Fontaine JM: Microvolt T-wave alternans and the risk of death or sustained ventricular arrhythmias in patients with left ventricular dysfunction. J Am Coll Cardiol 2006;47:456-463

26. Murata M, Harada M, Shimizu A, Kubo M, Mitani R, Dairaku Y, Matsumoto T, Matsuzaki M: Effect of long-term beta-blocker therapy on microvolt-level T-wave alternans in association with the improvement of the cardiac sympathetic nervous system and systolic function in patients with non-ischemic heart disease. Circ J 2003;67:821-825.

27. Bloomfield DM, Hohnloser SH, Cohen RJ: Interpretation and classification of microvolt T wave alternans tests. J Cardiovasc Electrophysiol 2002;13:502-512.

28. Hohnloser SH, Ikeda T, Cohen RJ: Evidence regarding clinical use of microvolt T-wave alternans. Heart Rhythm 2009;6(Suppl 3):S36-S44.

29. Chan PS, Stein K, Chow T, Fendrick M, Bigger JT, Vijan S: Costeffectiveness of a microvolt T-wave alternans screening strategy for implantable cardioverter-defibrillator placement in the MADIT-II-eligible population. J Am Coll Cardiol 2006;48:112-121. 